\title{
A framework for validating noninvasive genetic spatial capture-recapture studies for rare and elusive species
}

\author{
Samantha McFarlane ${ }^{1}$, Micheline Manseau ${ }^{2}$, Robin Steenweg $^{3}$, Dave Hervieux ${ }^{3}$, Troy \\ $\mathrm{Hegel}^{3}$, Simon Slater ${ }^{3}$, and Paul Wilson ${ }^{1,1}$ \\ ${ }^{1}$ Trent University \\ ${ }^{2}$ Environment and Climate Change Canada \\ ${ }^{3}$ Alberta Environment and Parks
}

April 28, 2020

\begin{abstract}
Accurately estimating abundance is a critical component of monitoring and recovery of rare and elusive species. Spatial capturerecapture (SCR) models are an increasingly popular method for robust estimation of ecological parameters. We provide a maximum likelihood analytical framework to assess results from empirical studies to inform SCR sampling design, using both simulated and empirical data from non-invasive genetic sampling of seven boreal caribou populations (Rangifer tarandus caribou) which varied in range size and estimated population density. We use simulated population data with varying levels of clustered distributions to quantify the impact of non-independence of detections on density estimates, and empirical datasets to explore the influence of varied sampling intensity on the relative bias and precision of density estimates. Simulations revealed that clustered distributions of detections did not significantly impact relative bias or precision of density estimates. The empirical genotyping success rate was $95.1 \%$. Empirical results indicated that reduced sampling intensity had a greater impact on density estimates in smaller ranges. The number of captures and spatial recaptures were strongly correlated with precision, but not relative bias. The best sampling designs did not differ with estimated population density but differed between large and small ranges. We provide an efficient framework implemented in $\mathrm{R}$ to estimate the detection parameters required when designing SCR studies. The framework can be used when designing a monitoring program to minimize effort and cost while maximizing effectiveness, which is critical for informing wildlife management and conservation.
\end{abstract}

\section{Key words}

density estimation; non-invasive genetic sampling; population estimation; precision; spatial capture-recapture; study design

\section{Introduction}

Robust abundance estimates are fundamental parameters for managing wildlife populations, and central to understanding extinction risk (Campbell et al., 2002; Lande, 1993; Shaffer, 1981). Monitoring and understanding variation in abundance is critical for recovery efforts of threatened and endangered populations; however, producing accurate population estimates remains a challenge for many species. This is particularly true for species that occur at low density or in low abundance, that are cryptic, or that exhibit elusive behaviours which make capture difficult (Kéry, Gardner, Stoeckle, Weber, \& Royle, 2011; Pollock, Marsh, Lawler, \& Alldredge, 2006). Non-spatial capture-recapture (CR) analyses have been the standard method 
used to estimate abundance of many vertebrate species, however, spatially-explicit capture-recapture (SCR) models are becoming the new standard because they are robust to small sample sizes, produce precise density estimates, and can accommodate low capture probabilities (Borchers \& Efford, 2008; Efford, Borchers, \& Byrom, 2009; Ivan, White, \& Shenk, 2013; Royle, Chandler, Sollmann, \& Gardner, 2013). By including spatial information of captured individuals directly into the analyses, SCR models resolve issues surrounding the effective trapping area and temporary migration and are robust to assumptions about geographic closure that are common issues in non-spatial CR studies (Efford \& Fewster, 2013; Royle et al., 2013). Recapturing individuals at different locations also provides information on individual activity centers, which are used to estimate animal density within the study area (Borchers \& Efford, 2008; Royle et al., 2013).

SCR models directly depend on adequate number of unique individuals captured and recaptured at multiple spatial locations (Efford \& Boulanger, 2019; Sun, Fuller, \& Royle, 2014). Simulations are recommended to enable the assessment of sampling design on SCR parameter estimates, to inform optimal sampling design (Royle et al., 2013). Such studies have primarily focused on large carnivores, such as black bears (Ursus americanus ; Clark, 2019; Sollmann et al., 2012; Sun et al., 2014; Wilton et al., 2014), and a few additional taxa (Kristensen \& Kovach, 2018; Tobler \& Powell, 2013), while limited work has been done on species occurring at low densities over large areas and with more limited home range sizes. Non-invasive genetic sampling approaches can be used to alleviate the challenges associated with surveying rare and elusive species, by constructing capture histories from DNA collected from feces, hair, or other non-invasively collected samples (Kristensen \& Kovach, 2018; Lampa, Henle, Klenke, Hoehn, \& Gruber, 2013; Waits \& Paetkau, 2005). Non-invasive methods often result in higher capture rates and lower expense than traditional capturerecapture methods (Lampa et al., 2013; Prugh, Ritland, Arthur, \& Krebs, 2005; Waits \& Paetkau, 2005), and SCR is increasingly being used in combination with non-invasive methods (Royle et al., 2013; Kristensen \& Kovach, 2018; Lamb et al., 2018). Knowledge of the target species' home range size helps inform the spatial sampling design (Sollmann et al., 2012; Sun et al., 2014), but empirical studies are still necessary as detection probabilities may be influenced by other factors (e.g. variable habitat conditions (Efford \& Fewster, 2013). Efford \& Boulanger (2019) presented formulae to determine the precision of new study designs by computing intermediate variables, such as the number of detected individuals and expected number of recaptures, which strongly correlate with precision. However, these formulae require starting values for density and detection parameters (Efford, 2019b), which may not be available for less studied species.

Here, we developed a framework to assess results from empirical studies to inform sampling designs (Fig. 1 ). The framework consists of (1) determining the number of unique individuals captured and spatially recaptured from empirical data; (2) running SCR models under the assumption of homogeneous distribution to estimate the detection parameters $g 0$ (detection probability) and $\sigma$ (spatial extent of an individual's use of the landscape) to assess the precision of the density estimates; (3) running simulations to assess the influence of the species' behaviour on density estimates and relative bias; (4) using empirical data to assess different sampling designs and evaluate precision and relative bias of the estimates; and (5) making recommendations on study design based on the resulting precision and relative bias of the estimates. The framework is implemented in R (R Core Team, 2019), using maximum likelihood methods.

To collect empirical data, we completed aerial surveys across the ranges of seven boreal caribou populations in Alberta, Canada. These ranges varied in size, exhibited differences in estimated caribou population density, and contained different levels of natural and anthropogenic disturbances (Fig. 2; see Appendix 1 for details). For each caribou population we used an aerial transect survey design to conduct non-invasive genetic sampling, through the collection of caribou fecal pellets. While we studied boreal caribou, our approach for evaluating study design is applicable to other species and systems. 


\section{Materials and Methods}

\subsection{Fecal pellet collection and genetic analysis}

For each population, we flew 3 surveys to collect fecal pellets during winter (December to March), with sampling occasions spaced approximately one-month apart. Following the aerial survey protocol outlined in Hettinga et al. (2012), aerial transects were systematically flown at 3-km intervals across each entire caribou population range using rotary- or fixed-wing aircraft, or a combination of both aircraft, to locate caribou feeding locations, for a total of 69,070 km flown across the seven ranges (Table 1). Once located, personnel landed at each feeding site and collected fecal samples; this included collecting samples from backtracking on caribou trails. All pellet samples were kept frozen at $-20^{\circ} \mathrm{C}$ until DNA extraction was performed.

In the laboratory, fecal samples were thawed and the mucosal coat surrounding the pellets was removed for DNA analysis. The extraction protocol used to amplify the DNA is outlined in Ball et al. (2007). Following quantification of target caribou DNA, samples were diluted down to a working stock concentration of $2.5 \mathrm{ng} / \mathrm{ul}$. We amplified the DNA at 9 variable fluorescently labelled microsatellite loci (FCB193, RT7, RT1, NVHRT16, BM888, RT5, RT24, RT6, OHEQ; Bishop et al., 1994; Cronin, MacNeil, \& Patton, 2005; Wilson, Strobeck, Wu, \& Coffin, 1997) to generate individual-specific genetic profiles, along with caribouspecific Zfx/Zfy primers for sex identification. The amplification protocol is outlined in Ball et al. (2007). Following amplification, each sample was genotyped on the ABI 3730 DNA Analyzer (Applied Biosystems) Microsatellite alleles were scored with the program GeneMarker v1.91@ (SoftGenetics, State College, PA) and followed a protocol documented in Flasko et al. (2017) and McFarlane et al. (2018). Unique individuals were identified using the program ALLELEMATCH (Galpern, Manseau, Hettinga, Smith, \& Wilson, 2012). We retained samples that amplified at [?]5 loci and re-amplified apparent unique genetic profiles represented by a single sample using two independent scorers to confirm unique individual identities (Hettinga et al., 2012). An error rate per locus was calculated using these re-amplification results.

\subsection{Framework}

\subsubsection{Empirical SCR modeling}

We used a maximum likelihood approach implemented in the R packagesecr (Efford, 2018; R Core Team, 2019 ) to estimate boreal caribou densities. SCR models are comprised of a submodel for the distribution of animals in the area of study (population density, $D$ ), and a submodel for the detection process, given the detection probability (the intercept of the detection function, g0 ) and given a parameter for scaling the detection function (the spatial extent of an individual's use of the landscape - $\sigma$; Borchers \& Efford, 2008; Efford et al., 2009). For our empirical data, we treated each survey as an occasion within a single session. We discretized the study area into a $1500 \mathrm{~m}$ grid of proximity detectors, (which record the presence of individuals at each detector without restricting movement; Efford et al., 2009). The area of integration for SCR models needs to be large enough such that animals residing beyond the study area have a negligible chance of being detected (Borchers \& Efford, 2008; Efford, 2004; Royle \& Young, 2008). We therefore defined our state-space with a $15 \mathrm{~km}^{2}$ buffer around all study areas. We ran models for females, males, and both males and females together.

We estimated the parameters of the SCR detection function ( $g 0$ and $\sigma$ ) by maximizing the conditional likelihood, and derived density $(D)$ from the top $\mathrm{AIC}_{\mathrm{C}}$-ranked models (Anderson, Burnham, \& White, 1994; Borchers \& Efford, 2008). We used the hazard exponential form of the detection function, because area search data models the cumulative hazard of detection (Efford, 2011). Models assumed that individuals were identified correctly, populations were demographically closed during sampling, and detections were independent and conditional on activity center (Borchers \& Efford, 2008; Efford, 2004). We assessed sources of variation on the detection parameters with time and behaviour effects on both $g 0$ and $\sigma$. 


\subsubsection{Testing assumptions of homogeneous distribution}

Boreal caribou is a non-migratory ecotype of caribou and have relatively small home ranges compared to wide-ranging carnivores such as brown bears (Graham \& Stenhouse, 2014; Lamb et al., 2018) and black bears (Whittington \& Sawaya, 2015). Boreal caribou group size fluctuates throughout the year; group size is lowest during spring and summer when cows become solitary for calving, increases before the rut, and peaks in early to late winter (Thomas \& Gray, 2002). To assess how the distribution of the animals (i.e. clustering) affected the precision and relative bias of our estimates, we simulated different population distributions using three of our empirical datasets (Little Smoky, Cold Lake, and Slave Lake). Different distributions can be used for the simulations including a homogeneous Poisson distribution, inhomogeneous or clustered Poisson distributions (Efford, 2019a). The chosen population distribution should reflect the distribution of the study species. Our empirical data approximated a Neyman-Scott clustered Poisson distribution which was then used for the simulations (Efford, 2019a). To simulate multiple detections in very close proximity, we set the spatial scale $(\sigma)$ of the 2-D kernel for locations within each cluster to be 1 . To simulate varying levels of clustering, we varied the fixed number of individuals per cluster (see Figs S1.1-S1.3). We selected starting values for $\mathrm{D}, \mathrm{g} 0$ and $\sigma$ from the empirical model runs (Table 2). We carried out all simulations in the secr R package (Efford, 2018; R Core Team, 2019).

\subsubsection{Assessing precision and relative bias of different sampling designs using empirical data}

We repeated the empirical population analyses with subsamples of data to explore how reduced sampling intensity affected the relative bias and precision of the density estimates from our empirical study. We rarified the data by reducing the number of sampling occasions and reducing the number of aerial transects flown. For the reduced number of sampling occasions, all possible 2-occasion combinations were run (occasions 1 and 2; occasions 2 and 3; and occasions 1 and 3). Aerial transects were removed from the original spatial field data, keeping either every second or third transect line to emulate sampling strategies of $6 \mathrm{~km}$ or 9 $\mathrm{km}$ transects. Only the samples collected along the remaining transect lines were retained, and only those detectors along the remaining transect lines were used in the analysis. We used the coefficient of variation $(\mathrm{CV})$ as the metric for precision, and calculated the relative bias $(R B=(\hat{D}-D) / D)$ as the metric for bias (as in Tobler \& Powell, 2013; Efford \& Boulanger, 2019; Efford \& Fewster, 2013; Kristensen \& Kovach, 2018). We compared estimates from the reduced datasets $(\hat{D})$ to those based on the empirical dataset $(D)$. We considered models with CV $<20 \%$ (following Pollock et al. 1990) and relative bias $<15 \%$ (Otis, Burnham, White, \& Anderson, 1978) as favourable outcomes. Models with CV $<30 \%$ and $\mathrm{RB}<20 \%$ can also be considered favourable (Kristensen \& Kovach, 2018), because high precision may be difficult to achieve for rare and low-density species.

We calculated the precision and relative bias of each subsampling scenario. Scenarios were considered precise when CV $<30 \%$ and RB $<20 \%$. To determine how the number of captures, number of recaptures, and number of spatial recaptures (recaptures at different locations) influence the precision and relative bias of the estimates, we correlated the precision and relative bias of the estimates with these parameters for each scenario, and then globally.

\section{Results}

\subsubsection{Capture and spatial recapture rates}

A total of 7,210 fecal samples were collected and 6,865 were successfully genotyped (average $95.1 \%$ genotyping success), resulting in the identification of 1,755 unique individuals from the seven populations (Table 1). Only

four allelic dropout amplification errors occurred (error rate $<0.001 \%$ ). We obtained adequate number of captures, number of unique individuals, number of recaptures, and number of spatial recaptures for all seven populations (Table 1), with the lowest spatial recapture rates being in the smaller populations of Little 
Smoky and Slave Lake. We had similar recaptures and spatial recaptures rates for females and males (Table S2.1, Table S2.3).

\subsubsection{Empirical model performance}

Density estimation for both sexes combined and for females had good precision ( $\mathrm{CV}<30 \%$; Table 2, Table S2.1), but modelling males separately led to density estimates for some populations having poor precision (Table S2.2). The average detection probability was low $(g 0<0.06$; Table 2) for all populations except the first sampling occasion for Slave Lake $\left(g 0_{t 1}=0.66, g 0_{t 2}=0.036, g 0_{t 3}=0.44\right) . \sigma$ differed among populations, ranging from 1,226 $\mathrm{m}$ in Slave Lake to 3,363 $\mathrm{m}$ in Cold Lake (Table 2).

\subsubsection{Assumptions of homogeneous distribution}

Results of simulations showed that clustering of caribou detections did not impact the precision or relative bias of the density estimates (Appendix 2). Median density estimates remained similar and slightly above the starting density for all levels of clustering density $(\mu)$ for the three simulated populations. The simulated Cold Lake population estimates retained the highest precision and were relatively unbiased, despite clustering, which corresponds with the precision found for the empirical model (Table 2). The simulated Little Smoky and Slave Lake population density estimates had lower precision than Cold Lake when caribou were clustered, but median density estimates were not affected by clustering, and density estimates from both populations remained unbiased (Appendix 2). Using a threshold value for precision of CV $<30 \%$, Little Smoky and Slave Lake had inadequate median levels of precision at all levels of $\mu$. These populations had similar (Little Smoky $\sigma=1600 \mathrm{~m}$ ) or smaller (Slave Lake $\sigma=1200 \mathrm{~m}$ ) $\sigma$ values compared to the chosen detector spacing of $1500 \mathrm{~m}$ (see Appendix 4). The detector spacing of $1500 \mathrm{~m}$ for the empirical studies for these populations was too wide relative to $\sigma$, with very few spatial recaptures of individuals (36 in Little Smoky, 38 in Slave Lake over three occasions), as the detector spacing was larger than $\sigma$.

\subsubsection{Precision and relative bias of reduced sampling designs}

In total, 36 different subsampling scenarios were run for each population, for a total of 252 models. Precision and relative bias were positively correlated for all sexes (both sexes $r=0.557, p<0.0001$, female $r=0.597$, $p<0.0001$, male $r=0.634, p<0.0001$ ), with decreasing precision (increased CV) and increasing relative bias (divergence from the estimate from the full dataset) with increased transect spacing and reduced number of occasions (Figs 3-4). Several scenarios failed to converge for Little Smoky and Slave Lake at $6 \mathrm{~km}$ and $9 \mathrm{~km}$ due to low numbers of individuals and no recaptures, resulting in 227 completed models. The Little Smoky and Slave Lake ranges are two of the geographically smallest ranges (Table 1; Fig. 2), and samples in these areas were clustered geographically (Fig. 2). The detection function scaling parameter $(\sigma)$ for the empirical data for Little Smoky and Cold Lake were smaller than the detector spacing of $1500 \mathrm{~m}$ and reducing the number of transects increased the detector spacing even further, leading to the detector spacing being significantly larger than the $\sigma$ estimates for these populations.

Precision of the subsampling scenarios were influenced by the number of unique individuals, number of recaptures, and number of spatial recaptures (Fig. 5). Precision was negatively correlated with the number of individuals, with precision decreasing with fewer captured individuals (Table S2.5, Fig. 5); all models that failed to run had no recaptures of individuals. The larger ranges of Cold Lake, ESAR, WSAR and Red Earth had more unique individuals than the smaller ranges of Little Smoky, Nipisi and Slave Lake (Fig. 5). When determining the influence of the number of individuals on model precision, all models with three occasions had adequate precision $(<30 \% \mathrm{CV})$ for both sexes in the larger populations. The number of unique individuals had a greater influence in the smaller ranges, leading to inadequate precision in Little Smoky, Nipisi and Slave Lake (Fig. 5), with no significant correlation between precision and the number of unique individuals in Slave Lake (both sexes) and Little Smoky males (Table S2.5). Precision was negatively correlated with the number of recaptures (Table S2.6) and spatial recaptures (Table S2.7), with lower precision in the smaller populations compared to the larger populations. All models with three occasions for the larger populations 
fell below the $30 \%$ CV threshold for all sex models (Fig. 5). Even when decreasing the number of occasions to two, the larger ranges still performed well with adequate precision, as these subsets still provided an adequate number of recaptures of individuals for the models to run and precision was significantly correlated to the number of recaptures (Table S2.6, Fig. 5). The smaller ranges did not perform as well when the data was reduced to two occasions; several models only retained one recapture of an individual, which resulted in a CV of nearly $100 \%$ (Fig. 5) and the number of recaptures or spatial recaptures was not significantly correlated with precision (Slave Lake both sexes, Little Smoky males, Slave Lake males; Table S2.6-Table S2.7).

While there was a strong relationship between precision and the number of individuals and recaptures, this was not the case for relative bias (Tables S2.5-S2.7; Fig. 5). Except for Nipisi (all sexes) and Red Earth females, the number of captures, number of unique individuals, recaptures or spatial recaptures was not significantly correlated with relative bias (Tables S2.5-S2.7). Removing the third session resulted in more bias compared to removing the first and second sessions (Fig. 6).

\section{Discussion}

We provide an efficient framework for estimating detection parameters required for SCR studies and validating empirical study designs for species where baseline detection data is not available. Our results using seven empirical datasets indicate that our genotyping protocol was highly successful, our capture and recapture rates were sufficient, and our study design was appropriate in producing precise and reliable density estimates. We followed the aerial survey protocol outlined in Hettinga et al. (2012) to inform our sampling design and obtained similar recapture rates between sampling occasions. We found that the detection parameters g0 (detection probability) and (the spatial extent of an individual's use of the landscape) varied among our study populations and between sexes (Table 2, Table S2.1, and Table S2.2). Our results were robust to reduced sampling intensity (both in frequency and spatially), with the best study design dependent upon range size, and not dependent upon estimated population density or the spatial distribution of individuals.

For multiple species, the SCR model assumption that animals are independently and uniformly distributed over a study area is often violated, as is the case for boreal caribou (Despres-Einspenner, Howe, Drapeau, \& Kuhl, 2017; Lopez-Bao et al., 2018; Stevenson et al., 2015). The fission-fusion social structure and dynamics exhibited by boreal caribou during the winter months leads to frequent exchanges between groups (Thomas \& Gray, 2002). Our simulation results show that SCR models performed reliably; the grouping and movement patterns of boreal caribou during our sampling period had minimal impact on the precision or relative bias of the density estimates. We found a slight overestimation in density estimates (Appendix 2), but the precision and relative bias were not impacted. Few studies have looked at the effect that non-independence of individuals has on SCR methodologies. Lopez-Bao et al. (2018) simulated scenarios of non-independence and spatial aggregation of individual wolves (Canis lupus) with only a slight underestimation in population abundance estimates of aggregated individuals, while Despres-Einspenner et al. (2017) were unsure to what extent the measures of uncertainty in their study of a community western chimpanzees (Pan troglodytes verus ) were underestimated.

Study designs can be inappropriate when poorly matched with the spatial behaviour of the target species (Williams et al., 2002). Detector arrays that are significantly smaller than one home range, or extreme detector spacing that leads to few or no spatial recaptures can result in biased SCR estimates (Efford, 2011; Efford \& Boulanger, 2019; Sollmann et al., 2012; Tobler \& Powell, 2013). Reducing the sampling intensity had a greater impact on populations with smaller range sizes regardless of density; reducing the number of transects flown led to extreme detector spacing with few or no spatial recaptures (Figs S4.4-S4.6). Increasing the temporal period of sampling can be an effective way of increasing the number of detected captures and recaptures available for analysis, which increases precision, however, increasing the temporal sampling period can also violate the assumption of population closure and lead to biased estimates (Dupont, Milleret, Gimenez, \& Bischof, 2019). We found that the effects of reducing the number of sampling occasions 
on density estimates was influenced by the timing of the survey. If resources were only available to perform 2 , rather than 3, sampling sessions, we recommend focusing on collecting samples early in the winter, rather than later in the winter, as we achieved relatively unbiased estimates $(\mathrm{RB}<20 \%)$ when retaining December, January, or February sampling occasions. Weather conditions during March surveys were not always favourable, with poor snow conditions and warm temperatures creating difficulties for finding animals and identifying fresh tracks and feeding areas.

Results from our empirical study provides a range of estimates that can be used for simulating surveys of boreal caribou in other locations. For poorly studied species, completing an initial empirical study is critical for obtaining accurate detection probability estimates. Due to the clustered, nonhomogeneous distribution of boreal caribou, extensive sampling of the entire population is recommended to ensure that clusters of caribou are not missed during sampling. Our subsampling scenarios showed how less extensive sampling in smaller ranges can miss a large portion of the population, increasing the relative bias and imprecision of the density estimates. Applying the same sampling design to all seven of our study populations proved to be suboptimal; detector spacing for the smaller populations relative to sigma led to imprecise estimates.

Our analytical framework allowed us to examine the results of empirical surveys in depth, providing confidence in the density estimates. Through different simulations we were able to explore how relative bias and precision of estimates vary when assumptions are violated. We showed that the number of individuals and recaptures of individuals can be used to predict precision, but that they cannot be used to predict relative bias. Efford \& Boulanger (2019) state that subsampling of data to emulate different configurations of detectors, or different temporal sampling can be prohibitively slow, due to model fitting being computer-intensive; however, we found that even for our largest population model $\left(24,737 \mathrm{~km}^{2}, 386\right.$ unique individuals, and 545 recaptures), modelling with time and behaviour effects on both $g 0$ and $\sigma$ ran relatively quickly $(\sim 7-10$ days on a high-performance computer cluster) in a maximum likelihood framework, where the density model was fitted by maximizing the conditional likelihood.

We recommend the combination of non-invasive DNA sampling, together with SCR modeling and distribution simulations, to be an effective, accurate and precise approach to monitoring wildlife.

\section{Acknowledgements}

We would like to thank Sonesinh Keobouasone for help with data management and coding; Bridget Redquest, Jill Lalor, and Austin Thompson at Trent University for the DNA extraction and analysis; David Iles for the manuscript review; and staff from the Government of Alberta for collecting field samples.

\section{Authors' contributions}

DH, MM, TH, RS and SS conceived and designed the study. SS and RS lead the surveys and sample collection. SM implemented the analysis with contributions from MM and RS. PW coordinated DNA extraction and analysis. SM wrote the first draft of the manuscript with help from MM. All authors contributed to subsequent drafts and gave final approval for publication.

\section{Data availability}

Data deposited in the Dryad Digital Repository (link )

Link for the analysis $\mathrm{R}$ code available at Github (link ). 


\section{References}

Anderson, D. R., Burnham, K. B., \& White, G. C. (1994). Model selection in overdispersed capture-recapture data. Ecology , 75 (6), 1780-1793. doi:10.2307/1939637

Ball, M. C., Pither, R., Manseau, M., Clark, J., Petersen, S. D., Kingston, S., .. Wilson, P. (2007). Characterization of target nuclear DNA from faeces reduces technical issues associated with the assumptions of low-quality and quantity template. Conservation Genetics , 8 (3), 577-586. doi:10.1007/s10592-006-9193y

Bishop, M. D., Kappes, S. M., Keele, J. W., Stone, R. T., Sunden, S. L., Hawkins, G. A., . . Yoo, J. (1994). A genetic linkage map for cattle. Genetics ,136 (2), 619-639.

Borchers, D. L., \& Efford, M. G. (2008). Spatially explicit maximum likelihood methods for capturerecapture studies. Biometrics , 64 (2), 377-385. doi:10.1111/j.1541-0420.2007.00927.x

Campbell, S. P., Clark, J. A., Crampton, L. H., Guerry, A. D., Hatch, L. T., Hosseini, P. R., .. O'Connor, R. J. (2002). An assessment of monitoring efforts in endangered species recovery plans. Ecological Applications ,12 (3), 674-681. doi:10.1890/1051-0761(2002)012[0674:AAOMEI]2.0.CO;2

Clark, J. D. (2019). Comparing clustered sampling designs for spatially explicit estimation of population density. Population Ecology , 61 (1), 93-101. doi:10.1002/1438-390X.1011

Cronin, M. R. A., MacNeil, M. D., \& Patton, J. C. (2005). Variation in mitochondrial DNA and microsatellite DNA in caribou (Rangifer tarandus ) in North America. Journal of Mammalogy , 86 (3), 495-505. doi:10.1644/1545-1542(2005)86[495:vimdam]2.0.co;2

Despres-Einspenner, M. L., Howe, E. J., Drapeau, P. \& Kuhl, H. S. (2017). An empirical evaluation of camera trapping and spatially explicit capture-recapture models for estimating chimpanzee density.American Journal of Primatology, 79 (7), e22647. doi: 10.1002/ajp.22647

Dupont, P., Milleret, C., Gimenez, O., \& Bischof, R. (2019). Population closure and the bias-precision tradeoff in spatial capture-recapture. Methods in Ecology and Evolution , 10 (5), 661-672. doi:10.1111/2041210X.13158

Efford, M. (2004). Density estimation in live-trapping studies. Oikos , 106 (3), 598-610. doi:10.1111/j.00301299.2004.13043.x

Efford, M. G. (2011). Estimation of population density by spatially explicit capture-recapture analysis of data from area searches. Ecology ,92 (12), 2202-2207. doi:10.1890/11-0332.1

Efford, M. G. (2018). Secr: Spatially explicit capture-recapture models. Retrieved from https://CRAN.Rproject.org $/$ package $=$ secr

Efford, M. G. (2019a).Package 'secr' . Retrieved from http://www.otago.ac.nz/density/pdfs/secr-manual.pdf

Efford, M. G. (2019b).Secrdesign miscellaneous tools . Retrieved from http://www.otago.ac.nz/density/pdfs/secrdesigntools.pdf

Efford, M. G., Borchers, D. L., \& Byrom, A. E. (2009). Density estimation by spatially explicit capturerecapture: Likelihood-based methods: Environmental and ecological statistics. In T. D. L., E. G. Cooch, \& M. J. Conroy (Eds.), Modeling demographic processes in marked populations (Vol. 3, pp. 255-269). Boston, MA: Springer. doi:10.1007/978-0-387-78151-8_11

Efford, M. G., \& Boulanger, J. (2019). Fast evaluation of study designs for spatially explicit capturerecapture. Methods in Ecology and Evolution ,10 (9), 1529-1535. doi:10.1111/2041-210X.13239

Efford, M. G., \& Fewster, R. M. (2013). Estimating population size by spatially explicit capture-recapture. Oikos , 122 (6), 918-928. doi:10.1111/j.1600-0706.2012.20440.x 
Flasko, A., Manseau, M., Mastromonaco, G., Bradley, M., Neufeld, L., \& Wilson, P. (2017). Fecal DNA, hormones, and pellet morphometrics as a noninvasive method to estimate age class: An application to wild populations of central mountain and boreal woodland caribou (Rangifer tarandus caribou ). Canadian Journal of Zoology , 95 (5), 311-321. doi:10.1139/cjz-2016-0070

Galpern, P., Manseau, M., Hettinga, P., Smith, K., \& Wilson, P. (2012). Allelematch: An R package for identifying unique multilocus genotypes where genotyping error and missing data may be present. Molecular Ecology Resources ,12 (4), 771-778. doi:10.1111/j.1755-0998.2012.03137.x

Graham, K., \& Stenhouse, G. (2014). Home range, movements, and denning chronology of the grizzly bear (Ursus arctos ) in West-Central Alberta. The Canadian Field-Naturalist, 128 (3), 223-234. doi:10.22621/cfn.v128i3.1600

Hettinga, P. N., Arnason, A. N., Manseau, M., Cross, D., Whaley, K., \& Wilson, P. J. (2012). Estimating size and trend of the North Interlake woodland caribou population using fecal-DNA and capture-recapture models. Journal of Wildlife Management, 76 (6), 1153-1164. doi:10.1002/jwmg.380

Ivan, J. S., White, G. C., \& Shenk, T. M. (2013). Using simulation to compare methods for estimating density from capture-recapture data. Ecology , 94 (4), 817-826. doi:10.1890/12-0102.1

Kery, M., Gardner, B., Stoeckle, T., Weber, D., \& Royle, J. A. (2011). Use of spatial capture-recapture modeling and DNA data to estimate densities of elusive animals. Conservation Biology , 25 (2), 356-364. doi:10.1111/j.1523-1739.2010.01616.x

Kristensen, T. V., \& Kovach, A. I. (2018). Spatially explicit abundance estimation of a rare habitat specialist: implications for SECR study design.Ecosphere , 9 (5), 1-17. doi:10.1002/ecs2.2217

Lamb, C. T., Mowat, G., Reid, A., Smit, L., Proctor, M., McLellan, B. N., .. Boutin, S. (2018). Effects of habitat quality and access management on the density of a recovering grizzly bear population. Journal of Applied Ecology ,55 (3), 1406-1417. doi:10.1111/1365-2664.13056

Lampa, S., Henle, K., Klenke, R., Hoehn, M., \& Gruber, B. (2013). How to overcome genotyping errors in non-invasive genetic mark-recapture population size estimation - a review of available methods illustrated by a case study. Journal of Wildlife Management, 77 (8), 1490-1511. doi: 10.1002/jwmg.604

Lande, R. (1993). Risks of population extinction from demographic and environmental stochasticity and random catastrophes. American Naturalist , 142 (6), 911-927. doi:10.1086/285580

Lopez-Bao, J. V., Godinho, R., Pacheco, C., Lema, F. J., Garcia, E., Llaneza, L., Palacios, V. \& Jimenez, J. (2018). Toward reliable population estimates of wolves by combining spatial capture-recapture models and non-invasive DNA montoring. Scientific Reports, 8 (1), 2177. doi:10.1038/s41598-018-20675-9

McFarlane, S., Manseau, M., Flasko, A., Horn, R. L., Arnason, N., Neufeld, L., Bradley, M., \& Wilson, P. (2018). Genetic influences on male and female variance in reproductive success and implications for the recovery of severely endangered mountain caribou. Global Ecology and Conservation, 16 , e00451. doi:10.1016/j.gecco.2018.e00451

Otis, D. L., Burnham, K. P., White, G. C., \& Anderson, D. R. (1978). Statistical inference from capture data on closed animal populations. Wildlife Monographs , (62), 3-135. doi:10.2307/3830650

Pollock, K. H., Marsh, H. D., Lawler, I. R., \& Alldredge, M. W. (2006). Estimating animal abundance in heterogeneous environments: An application to aerial surveys for dugongs. Journal of Wildlife Management , 70 (1), 255-262. doi:10.2193/0022-541X(2006)70[255:EAAIHE]2.0.CO;2

Pollock, K. H., Nichols, J. D., Brownie, C., \& Hines, J. E. (1990). Statistical inference for capture-recapture experiments. Wildlife Monographs , (107), 3-97.

Prugh, L. R., Ritland, C. E., Arthur, S. M., \& Krebs, C. J. (2005). Monitoring coyote population dynamics by genotyping faeces.Molecular Ecology , 14 (5), 1585-1596. doi: 10.1111/j.1365-294X.2005.02533.x 
R Core Team. (2019). R: A language and environment for statistical computing . Vienna, Austria: $\mathrm{R}$ Foundation for Statistical Computing. Retrieved from https://www.R-project.org/

Royle, J. A., Chandler, R. B., Sollmann, R., \& Gardner, B. (2013). Spatial capture-recapture . Waltham, MA: Academic Press.

Royle, J. A., \& Young, K. V. (2008). A hierarchical model for spatial capture-recapture data.Ecology , 89 (8), 2281-2289. doi:10.1890/07-0601.1

Shaffer, M. L. (1981). Minimum population sizes for species conservation. BioScience ,31 (2), 131-134. doi:10.2307/1308256

Sollmann, R., Gardner, B., \& Belant, J. L. (2012). How does spatial study design influence density estimates from spatial capture-recapture models? Plos One ,7 (4), 8. doi:10.1371/journal.pone.0034575

Stevenson, B. C., Borchers, D. L., Altwegg, R., Swift, R. J., Gillespie, D. M., \& Measey, G. J. (2015). A general framework for animal density estimation from acoustic detections across a fixed microphone array. Methods in Ecology and Evolution, 6 (1), 38-48. doi: 10.1111/2041-210X.12291

Sun, C. C., Fuller, A. K., \& Royle, J. A. (2014). Trap configuration and spacing influences parameter estimates in spatial capture-recapture models. PLOS ONE ,9 (2), e88025. doi:10.1371/journal.pone.0088025

Thomas, D.C., \& D.R. Gray. 2002. Update COSEWIC status report on the woodland caribouRangifer tarandus caribou in Canada, in COSEWIC assessment and update status report on the woodland caribou Rangifer tarandus caribou in Canada. Committee on the Status of Endangered Wildlife in Canada. Ottawa. 1-98 pp.

Tobler, M. W., \& Powell, G. V. N. (2013). Estimating jaguar densities with camera traps: problems with current designs and recommendations for future studies. Biological Conservation , 159 , 109-118. doi:10.1016/j.biocon.2012.12.009

Waits, L. P., \& Paetkau, D. (2005). Noninvasive genetic sampling tools for wildlife biologists: a review of applications and recommendations for accurate data collection. Journal of Wildlife Management ,69 (4), 1419-1433. doi: 10.2193/0022-541X(2005)69[1419:NGSTFW]2.0.CO;2

Whittington, J., \& Sawaya, M. A. (2015). A comparison of grizzly bear demographic parameters estimated from non-spatial and spatial open population capture-recapture models. Plos One , 10 (7), 17. doi:10.1371/journal.pone.0134446

Williams, B. K., Nichols, J. D., \& Conroy, M. J. (2002). Analysis and management of animal populations . San Diego, CA: Academic Press.

Wilson, G. A., Strobeck, C., Wu, L., \& Coffin, J. W. (1997). Characterization of microsatellite loci in caribou Rangifer tarandus, and their use in other artiodactyls.Molecular Ecology , 6 (7), 697-699. doi:10.1046/j.1365-294X.1997.00237.x

Wilton, C. M., Puckett, E. E., Beringer, J., Gardner, B., Eggert, L. S., \& Belant, J. L. (2014). Trap array configuration influences estimates and precision of black bear density and abundance. Plos One, 9 (10), 10. doi:10.1371/journal.pone.0111257

\section{Supporting Information}

Additional supporting information may be found online in the Supporting Information section at the end of the article. 


\section{Tables}

\section{Figures}

\section{Hosted file}

McFarlane_MER_manuscript_Table1.docx available at https ://authorea.com/users/309851/articles/ 440716-a-framework-for-validating-noninvasive-genetic-spatial-capture-recapture-studies-forrare-and-elusive-species

\section{Hosted file}

McFarlane_MER_manuscript_Table2.docx available at https://authorea.com/users/309851/articles/ 440716-a-framework-for-validating-noninvasive-genetic-spatial-capture-recapture-studies-forrare-and-elusive-species

\section{Hosted file}

McFarlane_MER_manuscript_Figure1.docx available at https://authorea.com/users/309851/articles/ 440716-a-framework-for-validating-noninvasive-genetic-spatial-capture-recapture-studies-forrare-and-elusive-species

\section{Hosted file}

McFarlane_MER_manuscript_Figure2.docx available at https://authorea.com/users/309851/articles/ 440716-a-framework-for-validating-noninvasive-genetic-spatial-capture-recapture-studies-forrare-and-elusive-species

\section{Hosted file}

McFarlane_MER_manuscript_Figure3.docx available at https://authorea.com/users/309851/articles/ 440716-a-framework-for-validating-noninvasive-genetic-spatial-capture-recapture-studies-forrare-and-elusive-species

\section{Hosted file}

McFarlane_MER_manuscript_Figure4.docx available at https://authorea.com/users/309851/articles/ 440716-a-framework-for-validating-noninvasive-genetic-spatial-capture-recapture-studies-forrare-and-elusive-species

\section{Hosted file}

McFarlane_MER_manuscript_Figure5.docx available at https://authorea.com/users/309851/articles/ 440716-a-framework-for-validating-noninvasive-genetic-spatial-capture-recapture-studies-forrare-and-elusive-species

\section{Hosted file}

McFarlane_MER_manuscript_Figure6.docx available at https ://authorea.com/users/309851/articles/ 440716-a-framework-for-validating-noninvasive-genetic-spatial-capture-recapture-studies-forrare-and-elusive-species 